\title{
DES IMAGES ET DES HOMMES
}

\author{
François Jost ${ }^{1}$
}

Deux écueils menacent les réflexions sur l'image. Le premier est derrière nous, mais la première sémiologie du cinéma vint s'y échouer : il fut de croire à une autonomie de l'objet, à la possibilité d'étudier celui-ci en dehors du champ de la communication et indépendamment de ses usages. La fin de la discipline (à la fois son but et sa mort) aurait été de parvenir au paysage complet de tous les codes requis pour faire un document audiovisuel, quels que fussent sa nature, son lieu de projection et l'attention spectatorielle. Dans cette perspective, la télévision n'était guère différente du film: "Film et télévision, en fait, ne sont rien d'autre que deux langages voisins qui

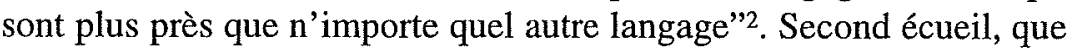
ne redoutent pas tous les navigateurs de la pensée, celui de réduire la diversité du visuel en fonction d'une axiologie (il y a l'image et le visuel, précisément) et de prétendre faire une théorie unifiante, dont l'universalité tiendrait à sa capacité de cerner l'essence de l'Image. Cette tentation de l'ontologie trouve ses racines dans la philosophie grecque et réactive, finalement, la question bazinienne du qu'est-ce que?

\footnotetext{
1 Professeur à l'Université de la Sorbonne Nouvelle-Paris III.

2 C. Metz, Langage et cinéma, Paris, Larousse, 1971, p. 179.
}

Recherches en communication, $\mathrm{n}^{\circ} 8$, (1997). 
Deux écueils que rencontrent des disciplines diverses (sémiologie, philosophie, etc.) mais qui, au fond, se réduisent à un seul : la transformation de l'image en une entité qui ne prend que faiblement son sens par rapport à son origine ou à sa destination humaines. Toutes les images se valent, aussi bien pour celui qui refuse d'accorder une place à l'évaluation dans les processus de cognition, que pour celui qui tente de mettre au jour leur "vraie" nature. Chez les narratologues, cette propension à l'autonomisation des entités visuelles est une conséquence de la crainte d'un autre danger: l'anthropomorphisation. Par peur d'y succomber, on multiplie les instances énonciatives en ayant soin d'éviter le problème de l'auteur.

\section{Les deux sources de l'image...}

A l'opposé, l'hypothèse qui conduit mon travail depuis plusieurs années est que la diversité des images et des documents ne peut s'expliquer que si l'on pose au départ que leur réception est soustendue par un anthropomorphisme régulateur. Pour qu'un film soit reçu comme une œuvre ou pour qu'un document soit reçu comme un discours sur la réalité, pardonnez ce truisme, il faut minimalement qu'il soit compris comme un artefact d'origine humaine et à destination humaine. Le document audiovisuel comme artefact commence là où le spectateur est capable de transfigurer la banalité du motif en un objet doté d'un "aboutness", comme dit A. Danto'. En cela le cinéma n'est différent ni de l'art moderne, et, notamment, des ready made, dont la naissance coïncide presque avec ses premières années, ni des mécanismes mis en jeu par l'interprétation littéraire. Si celle-ci ne requiert pas de la part de son lecteur de trouver l'intention réelle qui a présidé à sa conception et à sa fabrication -conception solidaire d'une théorie qui voit dans l'œuvre un processus de décodage symétrique de l'encodage- elle suppose, quoi qu'on en ait, une présomption d'intentionnalité.

Il n'est, pour s'en convaincre, que de considérer un instant ce qui se passe lorsque nous sommes en présence d'images produites sans intervention humaine, qu'il s'agisse de ces scènes d'horreur captées par des caméras automatiques placées dans des tranchées pendant la première guerre mondiale, de ces scènes quotidiennes que

1 A. DANTo, La tranfiguration du banal (trad. fr.), Paris, Éd. du Seuil, 1989. 
retransmettent un peu partout, dans les banques, les métros, les caméras de surveillance ou, encore, de ces portraits de gens qui lancent un message par l'intermédiaire de la télévision en défilant dans un camion équipé d'un dispositif d'enregistrement vidéo. Qu'observe le vigile d'aujourd'hui, qui, de son panopticon moderne, suit les allées et venues des passants? Que regarde le spectateur qui prend connaissance de l'annonce lancée par le passant par l'intermédiaire du petit écran?

Évidemment pas l'image, mais la réalité.

L'absence d'une présence humaine inférée derrière l'œilleton, déporte toute l'attention du spectateur sur l'objet enregistré : le gardien qui, assistant, grâce à ses écrans de contrôle, à un vol dans le supermarché qu'il est chargé de surveiller, se mettrait à commenter la qualité des cadrages, la qualité de la lumière d'une image ou le montage des plans générés par un processus aléatoire, sautant d'une caméra à l'autre, n'aurait assurément rien compris à la fonction du dispositif.

A la différence de l'image automatique, qui joue encore plus ou moins dans notre société comme une garantie d'authenticité (voir comment les images des caméras de surveillance du Ritz ont été proposées aux médias par la famille Al Fayed pour attester des derniers moments vécus par Dodi et Diana avant leur équipée tragique), l'image anthropoïde n'est qu'une promesse fondée sur la confiance accordée à la figure de l'énonciateur et chaque genre, comme je l'ai montré ailleurs ${ }^{1}$, se définit par une grande figure qui lui est associée. Promesse d'authenticité, le direct se fonde sur l'idée de témoin ou de narrateur-médiateur selon son degré de préparation. Promesse de lisibilité accrue du monde, le documentaire renvoie à la figure d'un manipulateur (sans les connotations péjoratives que l'on attribue à ce terme), qui construit l'espace et le temps en vue de leur intelligibilité. La fiction repose sur une promesse de pertinence narrative du visible et de l'audible dont on crédite le narrateur, tandis que l'œuvre d'art, pour être reçue comme telle, suppose que l'artiste organise images et sons en fonction d'une régulation esthétique.

Ces quatre grands masques anthropoïdes forment deux régimes fort différents séparés par une frontière radicale entre ceux dont le

\footnotetext{
1 Fr. Jost, "La promesse des genres", Réseaux, n 81, janvier-février 1997.
} 
médiateur est un Je-origine réel, comme dirait Käte Hamburger ${ }^{1}$, c'est-à-dire un Je qui renvoie à un sujet d'énonciation historique (témoin, manipulateur) et ceux dont le médiateur est un Je-origine fictif (narrateur, et, souvent, artiste). Il peut arriver qu'un reportage soit construit comme un film policier, avec son enquête, son dévoilement progressif de la réalité, ses rebondissements. Même s'il est un récit, il ne s'identifie pas à la fiction parce que nous croyons à l'existence d'un je-origine. Cet ancrage de la fiction dans le sujet de l'énonciation explique la grande disparité d'investissement anthropomorphe des figures que nous avons dégagées :

- Du côté de la fiction audiovisuelle, le narrateur implicite (non pas ce narrateur qui parle à visage découvert que j'appelle explicite) est toujours impersonnel ou anonyme. Son identité se réduit généralement à sa fonction : il ne devient manifeste qu'au moment où une intention narrative devient ostensible. Cette pauvreté d'investissement anthropomorphique est d'ailleurs observable dans les discussions concernant le reliable narrator: de façon générale, on imagine mal que le narrateur implicite mente, sans doute parce qu'il est la condition sine qua non de la communication narrative. En dernière instance, le manque de confiance en celui-ci est dû à une méfiance envers l'auteur, c'est-à-dire le Je-origine réel de l'œuvre ou du document, susceptible quant à lui de toutes les projections anthropomorphiques, puisque les médias -et surtout les magazines littéraires de la télévision- nous le présentent comme notre semblable, notre frère.

- Le magazine de reportage, en revanche, a ceci de particulier, que nous savons qu'il est énoncé par des Je-origines réels, ce qui donne prise à un imaginaire anthropomorphe. Vue sous cet angle, la question classique de la sémio-narratologie comment s'articulent image et son? devient la suivante : quelle identité tracent en creux les médiations visuelle et verbale ? Concourent-elles à la constitution d'une seule instance anthropomorphe ou de plusieurs? Quelles relations de savoirs les attachent l'une à l'autre ?

Cette interrogation est différente et complémentaire de celle de l'ocularisation, du point de vue oculaire, et de celle de la focalisation. Il ne s'agit ni de décrire les différents points de vue visuels ou sonores possibles, ni de penser l'articulation du savoir du narrateur avec celui du spectateur, mais de construire, pour ainsi dire, le degré de présence

1 K. Hamburger, Logique des genres littéraires [1957], trad. fr., Paris, Éd. du Seuil, 1986. 
du médiateur dans la réalité et son mode d'intervention dans la restitution du réel.

\section{Des images pour le dire}

Quel que soit le niveau de connaissance du téléspectateur sur la sociologie et l'histoire des professions télévisuelles, il remarquera, à condition d'y prêter un minimum d'attention, que la hiérarchisation de celui qui a la parole, le commentateur, si l'on veut, et de celui qui fait l'image est variable.

Parfois, le journaliste semble suivre comme nous la retransmission des images: par exemple, lors des championnats du monde d'athlétisme 1997, on put entendre un commentateur avancer que, pour savoir qui était le gagnant de telle course, il aurait fallu avoir une autre position par rapport à la réalité que celle restituée par l'écran de son moniteur de contrôle, qui était aussi le nôtre. A d'autres moments, le présentateur paraît en mesure de peser sur le contenu visuel en demandant, par exemple, au réalisateur de faire un zoom sur tel ou tel détail qu'il souhaite montrer au téléspectateur. Ces médiations verbale et visuelle s'incarnent dans des figures anthropoïdes de médiateurs -caméraman, journaliste- plus ou moins nommés : le premier est anonyme, le second connu du public. L'articulation d'une instance à l'autre pourrait avantageusement être pensée en termes de ce que Searle appelle la relation d'ajustement $d u$ mot au monde. On se souvient que, pour le philosophe américain, tout acte de langage revient à établir une relation vectorisée (ou asymétrique) entre le verbal et la réalité : les affirmations, les descriptions, les explications ont une direction d'ajustement qui va du mot au monde. Cet acte repose sur la recherche du mot juste pour caractériser un état du monde. Les demandes, les promesses vont du monde au mot, en ce sens qu'elles prescrivent un état du monde, de la réalité, qui doit se soumettre à l'autorité du verbal.

Le contexte visuel fourni par l'image animée modifie ces actes de trois façons.

1. Si l'acte de langage seulement verbal se définit d'abord par son intention, par l'attitude qu'y exprime le locuteur par l'intensité repérable dans des marqueurs de force illocutoire, l'image vient fournir une preuve ou une contre-épreuve immédiate aux dires. Alors que l'assertion, par exemple, repose d'abord sur le fait que le locuteur 
répond de ce qu'il affirme, qu'il doit être en mesure de fournir des preuves ou des raisons à l'appui de la vérité de la proposition exprimée', la simultanéité ou la consécution immédiate de l'image le décharge de cet exercice et l'accomplit à sa place. Illustrative ou non, l'image endosse le rôle dévolu dans la langue au locuteur.

2. Selon Genette, l'énoncé fictif peut être analysé "comme un acte illocutoire indirect (à mon sens large) et donc complexe, dont le véhicule est une assertion feinte ou non sérieuse, et dont la teneur est ad libitum une demande («Imaginez que...»), une déclaration ( $« \mathrm{Je}$ décrète fictionnellement que...»), voire une autre assertion, évidemment sérieuse, comme «Par la présente, je souhaite susciter dans votre esprit l'histoire fictionnelle d'une petite fille», etc."2. Dès le cinéma muet, les cartons mettent un terme à cette indétermination de la valeur de l'acte illocutoire de la fiction. Parce que la direction d'ajustement est quasiment attestée par la présence de l'image comme je viens de le dire, on sort de cette indifférence descriptive de l'énoncé fictif. Soit cet intertitre du Lys brisé (Griffith) :

\section{$\mathrm{n}^{\circ} 13$ Limehouse knows him only as a Chinese storekeeper ${ }^{3}$}

A première vue, il ne pourrait s'agir là que d'une proposition narrative, visualisée ensuite par la bande image, ou d'une description quasi scénaristique de l'image. Dès lors que l'on réfléchit sur l'acte qu'accomplit un tel carton, on doit bien admettre que l'énoncé pèse sur le spectateur de la scène plus qu'il ne décrit un état de fait. La représentation en plan général d'un carrefour où se trouve le magasin chinois, les trois personnes qui traversent l'écran et le parcours misérable de l'homme sont à lire comme la confrontation d'un immigré chinois à tout un quartier. La relation du carton à l'image n'a rien à voir avec l'opération structurale d'un ancrage qui arrêterait la dérive du sens, il "provoque une modification du statut ou de la situation de l'objet ou des objets auxquels il fait référence"4, ici visuellement, ce qui définit pour Searle un acte déclaratif.

Plus précisément, on peut même dire que ce type d'intertitre appartient à ces déclaratifs qui peuvent $s$ 'accomplir avec succès sans

1 J. SEARLE, Sens et expression, trad. fr., Paris, Éd. de Minuit, 1982, p. 105.

2 G. GenETTE, Fiction et diction, Paris, Éd. du Seuil, 1991, p. 56.

${ }^{3}$ Cf. "Les mots pour le voir", où j'analyse plus précisément le rôle de l'intertitre dans le cinéma muet (Imagine e scrittura nel cinema muto, Bologne, Clueb, 1998).

4 J. SeARle, op. cit., p. 57. 
le secours d'une institution extra-linguistique, comme les déclarations surnaturelles (du type Fiat lux) et les déclarations qui concernent la langue elle-même, comme "je nomme", "je désigne", "je définis", etc.

Contrairement à un performatif comme "je déclare la guerre", qui ne peut fonctionner que si le locuteur occupe une place donnée dans l'Institution, l'acte de nommer, dit le philosophe américain, n'exige aucune institution extra-linguistique. Néanmoins, en l'occurrence, l'acte déclaratif est soumis à la convention fictionnelle de la suspension de l'incrédulité, comme on le voit bien lorsqu'un carton indique Nuit, alors que le plan est manifestement tourné de jour : le spectateur doit alors accepter, pour que l'accomplissement de l'acte déclaratif réussisse, de conformer le monde au mot. Exceptés ces cas limites où le spectateur peut faire échouer la déclaration, le carton ne fonctionne évidemment que si le mot s'ajuste possiblement à l'image : même si un coin de rue ne s'identifie pas en général à la globalité d'un quartier, il doit être admis que "Limehouse" est un mot juste pour décrire la situation du monde narratif.

Le déclaratif a pour particularité d'ajuster à la fois le monde aux mots et les mots au monde. En tant qu'il décrète, il plie le monde à sa loi langagière ; en tant qu'accomplissement réussi, il décrit une modification de l'état du monde. Le carton partage cette ambivalence par rapport à la fiction visualisée : comme acte scriptural, il conforme l'image à ses mots (par la nomination et la définition des paramètres temporels, la qualification des actions dans l'image), il accomplit, en bref, une déclaration démiurgique; comme acte de lecture, il ne doit son succès qu'au fait que le spectateur trouve dans le monde profilmique les éléments conformes aux mots. En somme, le carton fictionnel est un acte déclaratif, parce qu'il fonctionne ainsi : s'il accomplit avec succès l'acte d'imposition et de nomination du sens à l'image, le monde a ce sens.

Cette dernière règle ne peut s'appliquer telle quelle quand il s'agit d'un récit factuel, car la portée du décret se trouve singulièrement restreinte. Tandis que la portée du déclaratif fictionnel est proportionnelle aux conventions qui le règlent (grâce auxquelles je peux accepter qu'un plan filmé de jour soit équivalent à une nuit), celle du déclaratif factuel se heurte à la fois à la connaissance spectatorielle du réel et à l'évaluation en termes de vrai et de faux dont relèvent les genres authentifiants. Que l'on me montre Paris le jour pour m'annoncer l'arrivée de tel homme politique la nuit dans la capitale française et je serai en droit de reprocher au journaliste de me 
mentir. Le commentaire ne peut être déclaratif qu'à deux conditions : la première est que le spectateur interprète plutôt en terme d'iconicité que d'indicialité, la seconde est qu'il trouve une communauté de sèmes entre la parole et le visuel; l'image d'archives remplit ces exigences pour autant qu'elle soit désignée comme telle : il est acceptable qu'on me montre n'importe quel cormoran englué dans du pétrole pour me décrire les effets de la marée noire, il ne l'est pas qu'on m'affirme que ce cormoran comme trace indicielle a été capté dans le golfe Persique, si ce n'est pas le cas. Dans le factuel, le déclaratif est évalué à l'aune de la nature annoncée du signe.

3. Tandis qu'un acte de langage dans un contexte linguistique engage son locuteur, et, par glissement, la plupart du temps le sujet empirique qui le prononce, dans un contexte audiovisuel surgit une interrogation sur la nature de ce locuteur. L'image est-elle là pour prouver ses dires ou règle-t-elle son propos à ce qui a été capté de la réalité ? Quel lien unit celui qui parle audiovisuel et celui qui est littéralement et non métaphoriquement doté d'une voix ? Dans le cas d'une configuration narratologique concernant un je-origine, comme c'est le cas ici, la réponse va dépendre en partie de l'hétéromorphie sémiotique du discours : la voix est une signature dans la mesure où par son grain elle renvoie à un corps singulier (Barthes); l'image peut aussi bien ne renvoyer à personne (nobody's shot) qu'à une entité empirique (identification primaire), qu'à une entité fictive (ocularisation interne primaire), même quand un document est globalement factuel.

\subsection{Au début était le verbe... L'image-esprit}

Ces observations en tête, nous tenterons de suivre l'écheveau intriqué des fils que tressent le commentateur, le filmeur et le motif du reportage (comme on dit travailler sur le motif en peinture).

L'explication journalistique à la télévision a un statut ambigu : en tant qu'acte de langage, sa direction d'ajustement va du mot au monde, elle tente de soumettre la parole à un schéma d'intelligibilité qui lui serait extérieur et qu'il s'agirait d'exprimer. Mais en tant qu'elle est restituée par l'image et le son ou illustrée, elle contraint l'image et donc la représentation du monde à sa loi. L'image doit s'ajuster à la parole explicative. Ainsi, le modèle de la transmission pure se caractérise par les traits suivants : l'image s'ajuste à la parole 
renversant purement et simplement la relation d'ajustement qui se serait produite sans elle; dans la mesure où elle se conforme à un schéma verbal préétabli, il arrive que la caméra précède le motif. Un exemple ancien, mais toujours actuel, celui de ce reportage de Cinq colonnes à la une sur la Rome olympique, où, après quelques vues touristiques de la Ville Éternelle -le Capitole, la via Appia, le Colisée, les thermes de Caracalla-, après des plans généraux du stade olympique en construction, on suit le trajet d'un homme à Vespa, qui se rend dans un café, pour nous faire comprendre ce qu'est le totocalcio. Une voix commente: "Nous allons vous l'apprendre en suivant M. Chicco"l.

Cette vectorisation verbo-iconique s'oppose à la description que donne à voir le commentaire d'un direct non préparé, une catastrophe, par exemple, où le journaliste tente en temps réel de conformer sa parole à l'image, de trouver le mot juste pour décrire ce qu'il voit, sans parvenir le plus souvent à sortir du pléonasme.

Une telle configuration est le degré zéro de l'anthropomorphisme. Savoir désincarné, le médiateur verbal est interchangeable et l'image impersonnelle. En d'autres termes, la parole du journaliste ne renvoie pas à un individu particulier, à un "sujet historique", mais à une "énonciation théorique", selon le mot de K. Hamburger, c'est-àdire une énonciation où l'individualité du sujet parlant n'est pas en cause, comme dans ces raisonnements géométriques où 1'on dit curieusement " $j e$ prends une droite, je trace une perpendiculaire", etc. alors que le raisonnement est universel ${ }^{2}$.

Même théorique, cette énonciation verbale conserve quelques caractères individuants. Notamment le sexe : la voix du savoir à la télévision fut pendant longtemps masculine ; en revanche, la médiation visuelle qui l'accompagne est généralement tout à fait désincarnée. Associée à un montage cinématographique en ocularisation zéro, la bande visuelle pose sur la réalité un regard distant, qu'on ancrerait à tort dans la personne du caméraman. A ce titre, beaucoup de reportages de Cinq colonnes à la une, même s'ils relèvent d'une idéologie

1 Reportage de Cinq colonnes à la une, 3 août 1959, Jean Prat et Raymond Marcillac.

2 Cf. K. Hamburger, Logique des genres littéraires, op. cit. Le lecteur de plus de 40 ans se souviendra peut-être du fameux sketch de Jean Yanne où un inspecteur commence une question à un candidat au permis de conduire de la façon suivante : "Vous êtes sur une route départementale..." et celui-ci de lui répondre avec entêtement: "Je prends jamais les routes départementales". La faute majeure du candidat est de confondre énonciation historique et énonciation théorique ! 
professionnelle qui identifie la réalité au terrain, et l'être au visible, sont bien loin de renvoyer à un ail, comme l'affirment Brusini et James $^{1}$ : plus souvent conformes à la rhétorique du montage classique, ils enchaînent des images-esprit dont on trouvera un exemple type dans ce reportage sur le pont de Tancarville (3 juillet 1959). Plongée sur le pont. Au niveau du sol, les voitures qui s'engagent sur celui-ci. Plan moyen d'un camion qui s'éloigne. Plongée sur l'embouchure de la Seine. Carte géographique pour situer Le Havre, etc. Comme dans n'importe quel film des années 50 , on procède par une décomposition analytique, de l'establishing shot aux plans à hauteur d'homme.

\subsection{Le temps d'un regard : l'image-corps}

L'ajustement de l'image par le mot, au lieu d'être guidé par une parole théorique qui reste extérieure au monde qu'elle décrit, peut venir de l'image elle-même. Cette fois, ce sont les sujets filmés -le motif- qui dictent, si ce n'est le choix des images, au moins leur pertinence informationnelle. Un exemple très typique : Les ados au jour le jour (A2, 18 juillet 1977, réal. M. Faillevic). L'émission débute sur un gros plan de jeunes qu'accompagne ce commentaire : " 14 au 30 juin 1976. Nous sommes venus dans ce square de la banlieue parisienne tous les jours de 16 heures à 22 heures. Nous y retrouvions un groupe de jeunes qui avaient l'habitude de se retrouver là". Après cette introduction lapidaire, le journaliste ne prendra plus la parole, si ce n'est à une ou deux reprises pour poser, off, une question à l'un ou à l'autre.

La vectorisation icono-verbale nous met dans une situation d'observation ethnographique assez proche de celle du "scaphandrier" que décrivait E. Morin à propos du documentaire "direct". Néanmoins, nul ne prétend ici que la caméra se fait (ou peut se faire) oublier : à un moment, deux adolescents demandent qu' on les filme en train de se battre ; à un autre moment, un jeune, assis sur un banc, se tourne vers l'objectif et lance au caméraman "Ça va, camarade ?" ; un enfant veut parler à la caméra, on fait le clap et on attend en vain sa déclaration, etc.

Alors que, dans les exemples de Cinq colonnes à la une que nous avons analysés, un fossé se creusait entre la formulation du monde par

1 ' $L$ 'œil, organe royal, soumet l'information télévisée à sa loi pendant plus de quinze ans", Voir la vérité, Paris, PUF, 1982, p. 63. 
le reporter (trouver le mot juste) et sa reformulation au spectateur (trouver l'image adéquate pour le mot), ici, la médiation visuelle est première : les soixante et onze minutes que nous voyons sont le résultat d'un "bain" de 96 heures dans la réalité. Cette différence de méthode de la restitution du réel recouvre une véritable rupture énonciative : dans le cas de la transmission d'un savoir désincarné, la suite des images est soumise à la parole et, donc, tout est fait pour invisibiliser la présence du filmeur : aussi, le reportage se construit-il sur une rhétorique de l'invisibilisation de l'énonciation audiovisuelle en se conformant aux codes en vigueur. "L'observation", quant à elle, est sous-tendue par l'idée que le "mieux que le sociologue puisse faire est d'objectiver les effets inévitables des techniques d'observation qu'il est obligé d'employer"'. L'accent est donc mis sur le tournage ou, plus généralement, sur la performance, entendue ici comme la prestation concrète d'un acteur de la communication audiovisuelle, animateur, journaliste, caméraman ou preneur de son. Cette emphase sur l'énonciation performantielle ${ }^{2}$ est un premier pas vers l'anthropomorphisation des images : même si le journaliste reste une voix off, même si le caméraman est dévoilé par une simple réplique qui lui est lancée, l'un et l'autre sont présents comme conditions transcendantales de la restitution du réel : de l'image-esprit, on se déplace vers une image-corps, une image incorporée. Les acteurs de la performance informative sont véritablement (et non théoriquement, comme dans Cinq colonnes à la une) dans une position de témoin oculaire. Position d'abord énonciative qui ne dote pas encore ces témoins d'un corps propre et, d'ailleurs, ne fait pas de ségrégation entre l'acteur de la médiation verbale (le journaliste) et ceux de la médiation audiovisuelle (caméraman ou preneur de son): bien que les questions viennent du journaliste, sa position ne l'extrait pas du reste de l'équipe, dont l'influence sur la réalité est globale.

1 P. Bourdieu, Questions de sociologie, Paris, Éd. de Minuit, p. 31.

2 Cf. F. Jost, "Quand y a-t-il énonciation télévisuelle?", in Penser la télévision, colloque dirigé par J. Bourdon et F. Jost, à paraître chez Nathan, 1998. 


\subsection{La somatisation de l'image}

Avec la fragmentation de l'équipe comme tout, s'ouvre la possibilité d'une anthropomorphisation des médiations verbale et visuelle et de leur autonomisation relative. Du côté de l'image, l'accent se déplace de l'appareil de prise de vue -pure position oculaire dans la réalité- à l'homme qui se trouve derrière l'oeilleton. C'est moins le tournage comme activité qui est révélé que la trace d'un corps dans l'espace filmé. Divers indices somatiques peuvent souligner que la caméra est une personne : mouvements accidentels (comme lorsqu'une balle perdue atteint l'objectif et que le cadre se renverse), mouvements intentionnels du cadreur qui cherche à transmettre le rythme, généralement effréné, de sa découverte ou de son avancée dans le monde ; angle de vue très marqué, renvoyant à une position ostensiblement intentionnelle. Ou, enfin, réaction des individus filmés face à la caméra. Je passe rapidement sur ces indices que j'ai étudiés ailleurs, non sans avoir noté qu'avec cette somatisation de l'image, la régulation de la direction d'ajustement verbo-iconique perd de son sens : si l'ordre des plans obéit encore -comme dans le modèle de l'explication- à l'ordre du discours verbal, la mise en cadre témoigne souvent d'un point de vue relativement autonome qui, en un sens, relève d'une autre logique, non plus argumentative, mais esthétique : de la traversée du réel, le journaliste retient un enchaînement temporel ou causal d'événements (par exemple, la descente de police dans un atelier clandestin), tandis que le filmeur, de son côté, cadre avec plaisir un motif purement visuel, plus attentif à la composition de son image et à son angle de prise de vue, au souci de faire un beau plan, qu'à la narration qui viendra la commenter.

\section{Le journaliste à visage découvert}

Du côté de la médiation verbale, la figure du témoin-narrateur prend corps quand celui-ci devient lui-même le motif du reportage. Plusieurs étapes sont décelables dans l'histoire de la télévision française. 


\subsection{Le reporter est une personne}

Première étape de cette incitation à l'anthropomorphisation : le journaliste est un individu identifiable. A "l'énonciation théorique" que représentait le commentaire désincarné se substitue une énonciation historique qui s'appuie sur la représentation d'un je-origine réel qui, loin de nous présenter la certitude d'un résultat, nous donne à voir ses hésitations et les méandres d'une pensée s'interrogeant sur la restitution du réel. Un bon exemple nous est fourni par Paule, émission de Claude Ventura extraite de la collection La saga des Français ${ }^{1}$. Le document, qui est globalement organisé selon la chronologie du journal intime (lundi, mardi, mercredi, etc.), fait se succéder deux types de séquences :

- l'une raconte, par l'intermédiaire d'une voix over, la rencontre hasardeuse d'un homme avec une infirmière. Bien qu'il porte le même nom dans le film et dans la vie ("Antoine" [Dulaure]), son identité et sa fonction ne sont guère plus assurées que dans un "nouveau roman" ("il écrit ; il est journaliste ou peut-être romancier ; il prépare un travail sur l'hôpital, une série d'articles, à moins qu'il ne s'agisse d'un roman. Il a choisi Beauvais, peut-être parce qu'il a été pensionnaire au lycée ou parce qu'un ami, à la suite d'un accident de la route, y a été hospitalisé ou parce que le nom même de la ville évoque une certaine vie de province ou peut-être pour toutes ces raisons à la fois (...) Au hasard de son enquête auprès de l'hôpital, il rencontre Paule...").

Dans cette première série discontinue, qui obéit à l'ordre du temps des rencontres et du quotidien, le spectateur observe à la fois la constitution d'une relation entre le sujet et l'objet de la quête, au sens d'une narratologie structurale, et une interrogation sur ce que parler du réel veut dire. Il ne s'agit plus, comme dans Rome olympique, de transmettre un savoir préexistant, mais de s'interroger sur les conditions transcendantales du reportage, ou, si l'on préfère, sur les conditions qui rendent possible la restitution d'un savoir -mieux : d'une expérience- de la réalité. Sont abordés successivement par la voix over, la nature du questionnement journalistique, la relation interviewer-interviewé, le rôle du contexte du tournage, la fonction sociale du journaliste, etc.

1 Série de M. de Castillo. Paule a été diffusée le 27 juin 1977 à 21 h55 (après La tête et les jambes). 
- la seconde série montre des images de l'hôpital : des malades qui parlent avec des médecins, ou qui passent sur un brancard dans un couloir, Paule qui s'occupe d'un vieillard. Deux procédés attestent qu'il ne s'agit à aucun moment de feintise ${ }^{1}$ : soit la caméra, observatrice distante, capte dans l'embrasure d'une porte la relation des malades et des soignants, soit elle accompagne -quasiment par-dessus l'épaule- Paule qui, sans y prendre garde, continue à exercer son métier.

Deux séries s'enlacent donc : l'une, qui met à distance le processus de l'enquête, par une confrontation de l'image (au sens le plus visuel du terme), du journaliste et du regard qu'il y porte, grâce à l'intériorisation de la voix over; l'autre, qui se déroule en dehors de lui, sans qu'il donne l'impression d'en être témoin, et qui montre la réalité quotidienne de l'hôpital. Le partage entre le temps de l'enquête, sa mise à distance et son enregistrement trace, délimite finalement, trois territoires, ou plutôt trois champs de souveraineté qui luttent ou interfèrent dans tout reportage : le point de vue idéologique du journaliste (répondant à la question : sous quel angle aborder la réalité ?), le point de vue visuel du caméraman (comment regarder le monde à travers l'œilleton d'une caméra ? Comment cadrer ?), et enfin, celui du réalisateur (comment faire pour que le spectateur épouse mon regard?).

Cette manière de leçon de perception par l'image et le son mêmes caractérise assez bien la télévision française des années 70 , encline à programmer des émissions qui jouent avec l'énonciation audiovisuelle pour former le regard spectatoriel, que ce soit sur le mode informatif que l'on vient de voir, sur le mode fictif (avec des séries comme Réalitélfiction ${ }^{2}$ ) ou sur le mode ludique (Télétest).

\subsection{Le journaliste est un personnage}

A la fin de cette décennie, cette interrogation adressée directement au médium se diégétise : la mise en avant de la quête d'un jeorigine réel, d'une personne, cède la place à celle, héritée de la fiction, du personnage de l'enquêteur, tel que nous le voyons mis en scène,

1 Sur ce concept, cf. F. JOST, "Le feint du monde", Réseaux, $\mathrm{n}^{\circ} 72-73$, juillet-octobre 1995.

2 Prod. : A2, J. Frappat. Entre le 10 avril 1977 et le ler mai 1977, les auteurs sont les suivants : J. Lavelli, A. Vitez, M. Mitrani, M. Rosier. 
par exemple dans Au prix d'une vie'. Comme dans Paule, le journaliste est devant la caméra et nous assistons partiellement à sa vie (i1 prend une chambre d'hôtel, se rase, mange, dort). Ici aussi, il met à distance les informations qu'il a recueillies (il écoute, comme Antoine, son magnétophone) mais l'enquête a un autre but. Il ne s'agit plus de restituer une "atmosphère", un mode de vie, mais d'élucider un comportement, de cerner la vérité d'un événement: le meurtre d'un adolescent qui semait la perturbation dans un bar, par un cafetier, acquitté par la suite sous les applaudissements du public du procès. Si, dans ce modèle de la compréhension, plutôt que de la transmission d'un savoir préalable, l'avantage est donné à la pluralité des points de vue (le journaliste interroge le cafetier, ses collègues, la police, des dresseurs de chiens, l'armurier, et, enfin, les amis de la victime), ce n'est plus sur le mode d'une certaine modernité qui mettrait en cause l'idée même de représentation, mais sur celui du policier qui reconstitue un faisceau de causes et d'effets à partir des éclats de réalité que constituent les témoignages. Somme toute, le journaliste étale devant nous ce qu'une instruction doit faire en secret et il se glisse donc dans le costume du policier (la voix over commente : "un certain nombre de points commencent à m'intriguer et je me procure le dossier de l'affaire; l'hypothèse d'une liaison entre Silas Bastien et madame Schneider ne résiste pas à l'examen des procès-verbaux" [26 minute]. Et le journaliste-policier d'insister sur les pièces qui présentent l'accusé sous un jour peu avantageux (raciste, petit bourgeois préoccupé par son image de patron de café). Bien que la figure du policier-enquêteur soit au service d'une conception plurielle de la vérité, ce n'est plus la médiation visuelle ou verbale qui se trouve mise en cause mais le modèle narratif du reportage porteur d'un résultat (identifié au savoir) et non d'un processus assimilé à la compréhension. En même temps, ce que pouvait avoir d'aride l'interrogation des codes audiovisuels par Paule est récupéré du côté du spectacle par une forme narrative inusable: l'enquête. Il est fort intéressant de constater que la responsable de ce programme (P. Breugnot) adopte, dans le cadre d'une télévision publique, un point de vue qui profite à la victime, qui tend à la réhabiliter tout en prenant partie contre 1'auto-défense, au moment où la Gauche, nouvellement installée au pouvoir, prenait de semblables positions.

1 Collection Mceurs en direct, Unité de programme P. Breugnot, réal. C. Massot, sc. C. Massot et P. Benquet, diffusion sur A2, le 3 octobre 1982. 
D'autant plus que cette même productrice, sous le règne de la Droite, et de la télévision privée qui en sera proche, concevra une émission, Témoin $n^{\circ} 1$, qui remettra constamment en cause la chose jugée et transformera les accusés en victimes dès lors que ceux-ci auront incarné des valeurs partagées par l'électorat d'une droite extrême (comme le droit à l'auto-défense).

\subsection{Le journaliste est une personnalité}

A ces témoins distancés, personne ou personnage, qui s'interrogent sur la représentation d'une vie ou sur le sens d'un événement pourra se substituer, dans les années 90, l'image d'un médiateur verbal qui soumet le visuel à la loi de sa profession: reporter. Deux mots qui forment le titre d'une émission diffusée sur $\mathrm{TF} 1^{1}$. Aux personnages sans qualité des deux programmes précédents, celui-ci oppose la personnalité médiatique de Marine Jacquemin qui, quelques semaines auparavant, a fait partie des quatre interviewers d'une prestation exceptionnelle du Président de la République française. A première vue, ce reportage sur les talibans de Kaboul, qui fonde sa légitimité sur un titre de film d'Antonioni, interroge la prétendue transparence des images en présentant au spectateur le contexte du tournage, comme dans Les Ados au jour le jour: les techniciens mettent en place leur matériel, la journaliste se soucie de trouver une autorisation de tournage, elle joint Paris par satellite, etc. Loin de jeter le soupçon sur la possibilité de restituer le réel, cette apparente mise en avant de l'énonciation audiovisuelle est d'abord chargée d'esquisser une sociologie de la profession : un reportage serait produit par un groupe humain fortement hiérarchisé en fonction d'une répartition de l'intelligible et du sensible : d'un côté, la journaliste, qui incarne le savoir, qui trace le chemin et explique aux techniciens la réalité qu'ils traversent; de l'autre, le caméraman, qui n'est qu'un prolongement de l'œil, un peu comme, pour Aristote, la main est le prolongement du cerveau. On constate comment cette idéologie du voir, chère aux professionnels de Cinq Colonnes, s'appuie sur une séparation des pouvoirs à l'intérieur de l'équipe, qui ne peut être mise en péril que lorsque ce sujet oculaire vide, simple instrument au service d'une pensée, prend véritablement forme humaine, c'est-à-

1 Diffusion le 28 avril 1997 à 22 h25. 
dire quand la présence de celui qui tient la caméra se fait trop forte. Deux cas de figure se rencontrent dans Profession reporter. Le premier surgit à l'occasion du filmage éventuel d'une cérémonie de mariage à laquelle les hommes n'ont pas le droit de participer. Seule autorisée à vivre ce moment, Marine Jacquemin apprend du caméraman le B.A. BA du filmage vidéo, au cours d'une scène où le cloisonnement des professions est réaffïmé avec insistance, en même temps que l'abandon de la rhétorique de l'image-esprit : derrière la caméra, il y a des hommes... ou des femmes et l'existence de l'image peut tenir à cette alternative. Deuxième cas de figure : ni hommes ni femmes ne sont admis à filmer. Cette situation se produit quand l'équipe parvient à Kaboul. "Se pose immédiatement le problème de l'image, dit Marine Jacquemin: cachée, volée, autorisée". Finalement, il est décidé de continuer le reportage en passant la caméra sous le manteau (elle est cachée dans le pardessus du caméraman qui justement, face au ministre du vice et de la vertu, semble être un simple journaliste). Commencé sous le signe de la médiatisation humaine du reporter et de son équipe, le magazine s'achève aussi sur l'idée que l'image la plus authentifiante est celle où la caméra prend la place de l'œil ("où est la caméra ?", demande Marine Jacquemin à celui qui l'a dissimulée dans ses vêtements). Mais cette prise de vue hasardeuse et aléatoire, au moins en ce qui concerne son cadre, n'a plus rien à voir avec les images automatiques qui ont été au point de départ de ma réflexion. Tout simplement, parce que leur fabrication comme leur réception sont subordonnées à une intentionnalité humaine explicitée par la médiation verbale. Montrer, c'est témoigner. Ce reportage repose évidemment sur un sophisme. Car tout en invitant le spectateur à lier la morale ou l'éthique du visuel aux motivations humaines qui sont à sa source et, même, aux individus qui en sont la cause matérielle, il ne s'interroge à aucun moment sur la façon dont nous sont communiquées les images du caméraman, du journaliste et du preneur de son... L'instance audiovisuelle ultime, est celée ou laissée dans l'ombre, ce qui accrédite encore l'hypothèse de ce pur esprit qui voit et capte la réalité, grâce à un objectif qui n'est même pas un œil...

On ne s'étonnera pas qu'une telle émission soit diffusée sur une chaîne privée : I'avantage donné ici à la femme-journaliste qui finit par étendre son champ de souveraineté de l'intelligible au visible permet évidemment, mieux qu'un reportage tourné uniquement vers le motif, de rendre efficaces les messages publicitaires. Comment 
juxtaposer le sort du peuple afghan et celui des consommateurs modernes que nous sommes? Par la figure humaine d'un être qui nous ressemble et que sa profession éloigne parfois d'un confort publicitaire qui pourrait cependant constituer son idéal : la suite des spots qui "trouent" l'émission sont à la fois l'envers et la continuité de l'univers que parcourt devant nous Marine Jacquemin : voiture climatisée Peugeot, pantalons américains Dockers, femme qui arrête de fumer (Nicorette), qui "craque" pour un homme "Brut", etc.

La médiatisation de la mort de Diana a posé de façon exemplaire cette question de l'anthropomorphisme des images et a déplacé le débat vers la source. A quelques jours d'intervalle, les images privées ont été condamnées quand elles venaient des paparazzi qui voulaient saisir l'image de la princesse à travers les vitres d'une voiture, approuvées quand il s'agissait de montrer Charles quittant l'hôpital de La Salpêtrière dans sa limousine et, même, saluées avec enthousiasme quand le père de Dodie $\mathrm{Al}$ Fayed présenta aux télévisions du monde entier les dernières vues de son fils et de Diana prises par des caméras automatiques de l'hôtel Ritz. Cette variation dans l'appréciation des images tendrait à prouver que les images nous intéressent beaucoup moins que ceux qui les font et incite à se poser une ultime question. Préfère-t-on être surveillés par des machines plutôt que par des hommes ? La question serait à méditer. 